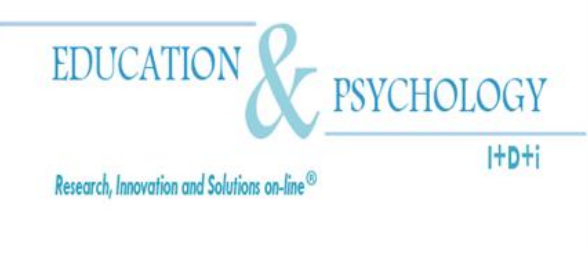

\title{
Concepciones de los estudiantes que ingresan en la Universidad, sobre el aprendizaje mediado por la lectura, en contextos de escritura académica
}

\section{Yanina Boatto ${ }^{1}$, Gisela Vélez ${ }^{2}$, Adriana Bono ${ }^{3}$ y Ana Vianco ${ }^{4}$}

${ }^{1,2,3}$ Facultad de Ciencias Humanas, Universidad Nacional de Río Cuarto.

${ }^{4}$ Facultad de Ciencias Económicas, Universidad Nacional de Río Cuarto.

\section{Argentina}

Correspondencia: Yanina Boatto. Ruta Nacional 36, Km. 601. Facultad de Ciencias Humanas. Universidad Nacional de Río Cuarto. Río Cuarto. Córdoba. Argentina (5800).

E-mail: yboatto@hum.unrc.edu.ar

(C) Education y Psychology I+D+i and Editorial EOS (Spain) 


\section{Resumen}

Introducción. En este trabajo se describen y analizan las concepciones de aprendizaje, a partir de la lectura de textos académicos que mantienen estudiantes universitarios, en la situación de leer para escribir junto a pares. El marco teórico se apoya en investigaciones sobre las concepciones de aprendizaje, entendidas como teorías implícitas (directa, interpretativa y constructiva) y en enfoques científicos de lectura (habilidades, interactivo y transaccional).

Método. El estudio asume un diseño no experimental transeccional descriptivo. Los participantes son estudiantes argentinos que ingresan a la universidad. Para la recolección de datos utilizamos un cuestionario de dilemas. Se procedió a analizar los datos con estadísticos descriptivos y correspondencias múltiples, en base a cuatro categorías (concepto, condiciones, procesos y resultados de aprendizaje a partir de la lectura) y tres dimensiones de análisis (reproductiva-directa, interpretativa-interactiva y constructiva-transaccional).

Resultados. Las respuestas de los estudiantes, respecto del concepto, se distribuyen de manera similar entre las teorías interpretativa-interactiva y constructiva-transaccional; respecto de las condiciones, se vinculan mayoritariamente con una teoría reproductiva-directa; en relación a los procesos, se reparten similarmente entre la teoría reproductiva-directa, la interpretativainteractiva y la constructiva-transaccional; en cuanto a los resultados, se vinculan mayoritariamente con una teoría constructiva-transaccional. En tanto que, las configuraciones que asumen estas categorías conformando concepciones, no se corresponden completamente con la estructura conceptual de las teorías implícitas de aprendizaje, descriptas en las investigaciones que constituyen nuestro marco empírico-conceptual.

Conclusión. Los resultados muestran que las concepciones estudiadas son inconsistentes, si se las compara con las teorías implícitas de aprendizaje. Las diferentes configuraciones conformadas se caracterizan por: a) ser homogéneas pero incompletas categóricamente en términos teóricos, o b) ser completas categóricamente pero heterogéneas en términos teóricos, en comparación con las teorías implícitas de aprendizaje.

Palabras Clave: concepciones de aprendizaje, lectura, escritura académica, estudiantes universitarios. 


\title{
First year university students: a study on their beliefs about learning through reading in an academic writing context
}

\begin{abstract}
Introduction. In this paper is describe and analyze the conceptions of learning from reading academic texts that keep college students, freshmen to education science, in the situation of reading to writing with peers. The theoretical assumption is supported by research on conceptions about learning, understood as implicit theories (direct, interpretative and constructive) and scientific approaches to reading (skills, interactive and transactional).
\end{abstract}

Method. The study assumes a non-experimental design, transactional descriptive. Participants are Argentine university students, freshmen to different areas of educational knowledge. For data collection was a questionnaire of dilemmas, built, tested, reviewed and validated for this research. The data analysis was performed by calculating descriptive statistics and multiple correspondence analysis, based on four categories (concept, conditions, processes and results of learning from reading) and three dimensions analysis (reproductive-direct, interpretativeinteractive and constructive-transactional).

Results. The answers of the students on the concept, is distributed similarly between interpretive-interactive and constructive-transactional theories; regarding the conditions, are mostly linked to a reproductive-direct theory; in relation to processes, are distributed similarly between direct-reproductive, interactive-interpretative and constructive-transactional theory; respect of results, mainly linked with one constructive-transactional theory. On the other hand, the configurations that assume these categories forming conceptions, not fully correspond to the conceptual structure of implicit theories of learning, described in investigations that constitute our empirical and conceptual framework.

Conclusion. The results obtained show that the conceptions studied are inconsistent when compared with the implicit theories of learning. Different shaped configurations are characterized by: a) being homogeneous but categorically incomplete in theoretical terms or b) being categorically complete but heterogeneous in theoretical terms, compared with the Implicit Theories about learning.

Key words: conceptions of learning, reading, academic writing, university students.

Received: 06/16/12 Initial acceptance: 09/04/12 Final acceptance: 11/15/12 


\section{Introducción}

Este trabajo -que es parte de una investigación mayor- se propone describir y analizar las concepciones de aprendizaje a partir de la lectura de textos académicos, en la situación de leer para escribir junto a pares, que mantienen los estudiantes que ingresan a la universidad, específicamente a ciencias de la educación.

El marco teórico se apoya en teorías constructivas, que otorgan valor al fuerte papel mediador que ejercen las concepciones de los sujetos en el aprendizaje de diversos dominios del conocimiento. Se atiende específicamente a investigaciones sobre las concepciones de aprendizaje entendidas como teorías implícitas. Las teorías implícitas se definen en base a su función fundamentalmente adaptativa y pragmática; los sujetos las activan en situaciones concretas y a partir de ellas actúan para dar respuestas a demandas y necesidades. De este modo, el análisis de las prácticas permitiría acceder al conocimiento de las teorías implícitas que mantienen los sujetos, ya que éstas difícilmente se exponen de modo declarativo (Atkinson y Claxton, 2000; Castorina, Barreiro y Toscano, 2007; Pozo y Gómez Crespo, 1998; Pozo y Scheuer, 1999; Pozo, Scheuer, Mateos y Pérez Echeverría, 2006; Rodrigo, Rodríguez y Marrero, 1993).

Interesa, particularmente, atender a las concepciones implícitas sobre el aprendizaje en el dominio de la lectura y la escritura, que mantienen los estudiantes que ingresan a la universidad. El interés por estos dominios del conocimiento se justifica en tanto que, como lo vienen señalando numerosas investigaciones, el ingreso a la universidad implica, entre otras cuestiones, el encuentro con nuevas tipologías textuales y exigencias de lectura y escritura, que demandan la activación de procesos psicológicos complejos y el aprendizaje de nuevas competencias (Carlino, 2005; Castelló, 2007; 2009; Fernández y Carlino, 2010; Fernández, Izuzquiza, Ballester, Marrón, Eizaguirre y Zanotti, 2010). Es así que la comprensión y producción de textos académicos por parte de los estudiantes de nivel superior, se ha instalado como un tema relevante en la agenda de investigación en psicología educacional de las últimas dos décadas.

Se han tomado como referentes empírico-conceptuales investigaciones actuales que se proponen indagar y conocer las concepciones implícitas de aprendizaje de los estudiantes universitarios, en el dominio de la lectura y la escritura, bajo el supuesto de que estas concepciones serían orientadoras de las acciones de los sujetos en situaciones de aprendizaje (Bosch y Scheuer, 2006; Pérez Echeverría, Mateos y Scheuer, 2006; Pozo y Pérez Echeverría, 2009; 
Vélez, 2006). Estas investigaciones forman parte de estudios más amplios, que se desarrollan en diversos dominios del conocimiento y en diferentes niveles educativos (Pozo y Scheuer, 1999; Pozo, Scheuer, Pérez Echeverría, Mateos, Martín y de la Cruz, 2006; Rodrigo, Rodríguez y Marrero, 1993).

Los estudios referidos a las teorías implícitas sobre el aprendizaje en distintos dominios del conocimiento, han descrito tres configuraciones denominadas Teoría Directa, Teoría Interpretativa y Teoría Constructiva. Estas teorías presentan características peculiares referidas al modo en que los sujetos entienden a las condiciones, los procesos y los resultados del aprendizaje, en base a la relación que establecen con los objetos de conocimiento en un dominio específico (Pozo, 2009; Pozo y Scheuer, 1999; Pozo, Scheuer, Mateos yPérez Echeverría, 2006; Rodrigo, Rodriguez y Marrero 1993).

Atendiendo al objeto de estudio de este trabajo, concepciones de aprendizaje a partir de la lectura, se vinculan conceptualmente estas relaciones entre sujeto y objeto de conocimiento, planteadas desde las teorías implícitas sobre el aprendizaje, con las relaciones entre lector, texto y contexto explicadas desde tres enfoques científicos de lectura: Lectura como Conjunto de Habilidades, Enfoque Interactivo y Enfoque Transaccional (Braslavsky, 2005; Dubois, 1987; Goodman, 1994; Rinaudo, 1999; Rosenblatt 1994) ${ }^{1}$.

Los estudios que describen a las teorías implícitas sobre el aprendizaje (Pozo, 2009; Pozo, Scheuer, Mateos y Pérez Echeverría, 2006) nos muestran que los sujetos que mantienen una Teoría Implícita Directa asumen una relación lineal entre las condiciones y los resultados del aprendizaje. Para ellos, bastaría con la co-presencia del sujeto y objeto de conocimiento para que se produzca el aprendizaje, entendido como un estado de conocimiento; además, consideran que el resultado obtenido debe ajustarse al objeto. En un sentido similar, desde el enfoque científico de la Lectura como Conjunto de Habilidades, se considera que el lector avanza en la lectura a partir de unidades del lenguaje, entendiendo al texto como un conjunto de signos. Así, va reconociendo lo que 'dice' el autor por medio de la decodificación y el resultado del aprendizaje se refleja en la reproducción literal de lo leído. De este modo, se producirían actos mecánicos de lectura y la comprensión del texto sería una consecuencia 'natural’ de la decodificación (Braslavsky, 2005; Dubois, 1987; Rinaudo, 1999).

Los sujetos que presentan una Teoría Implícita Interpretativa reconocen la interacción entre el sujeto y objeto de conocimiento por medio de la actividad mental del aprendiz, lo que 
da lugar a transformaciones del objeto; pero, al igual que el grupo anterior, creen que los resultados deben ajustarse al objeto de conocimiento (Pozo, Scheuer et al., 2006; Pozo, 2009). En vinculación con esta idea, desde el Enfoque Interactivo de Lectura, se entiende que el lector no recepta unidades aisladas del texto, sino que procesa estructuras o unidades mayorantes, significando las palabras y construyendo una representación mental del texto que se va modificando a medida que avanza en la lectura. El aprendizaje es el significado que 'se extrae' del texto, a partir de la apropiación del 'pensamiento' del autor; si bien se trata de intervenir para comprenderlo y desentrañar por lo tanto 'lo que quiere decir' el autor, se busca una única idea posible. De este modo, se desarrollaría un proceso de comprensión activo pero reproductivo (Braslavsky, 2005; Dubois, 1987; Rinaudo, 1999).

En cambio, los sujetos que se ubican en la Teoría Implícita Constructiva entienden al aprendizaje como un proceso de construcción y reconstrucción de las representaciones del mundo y de sí mismos acerca del mundo, advirtiendo que los procesos que median entre condiciones y resultados promueven transformaciones tanto de ellos mismos como del objeto de conocimiento, situación que se da en un contexto determinado que influye en dicho aprendizaje; si bien no ignoran al objeto, consideran que los resultados pueden asumir diferentes versiones (Pozo, Scheuer, Mateos y Pérez Echeverría, 2006). En esta misma línea de pensamiento, desde el Enfoque Transaccional de Lectura, la comprensión se considera activa e inferencial, entendiéndose que se da a partir del intercambio de ideas entre lector y autor, mediados por el texto, en un contexto determinado. Las características personales, los propósitos del lector y el momento particular de lectura hacen que cada sujeto pueda construir un significado personal, aun cuando se tengan en cuenta los referentes del texto y del contexto para validar las interpretaciones. Así mismo, la construcción del significado es diferente según el tipo de texto, su variedad discursiva y su estructura (Braslavsky, 2005; Dubois, 1987; Rinaudo, 1999).

\section{Objetivos}

Los objetivos de este trabajo son describir y analizar las concepciones de aprendizaje a partir de la lectura de textos académicos, en la situación de leer para escribir junto a pares, que mantienen los estudiantes que ingresan en la Universidad, específicamente a ciencias de la educación.

\footnotetext{
${ }^{1}$ Se apela a las teorías científicas como marco de referencia inicial para el análisis de las teorías personales (Correa y Camacho, 1993).
} 


\section{Método}

\section{Participantes}

Los participantes de la investigación constituyen la totalidad $(\mathrm{N}=118)$ de estudiantes universitarios que ingresan a diferentes áreas de conocimiento del campo de las Ciencias de la Educación (Psicopedagogía y Educación Especial), de la Facultad de Ciencias Humanas, de la Universidad Nacional de Río Cuarto, Argentina. El 95\% de los sujetos se conforma por mujeres que presentan una edad promedio de 17-18 años. La elección de este grupo de estudiantes se fundamenta en que ellos participan de un área de formación en la que la lectura y la escritura de textos académicos cobran particular relevancia.

\section{Instrumento}

Como se expuso en la introducción de este trabajo las teorías implícitas difícilmente cobran un carácter explícito; es por ello que sus características y supuestos se infieren a partir de las actuaciones y decisiones de los sujetos, de tal modo que para su estudio se requiere apelar a métodos de investigación indirectos. En ese sentido, investigaciones recientes dan cuenta de que los cuestionarios de dilemas son instrumentos de carácter estructurado adecuados para indagar este tipo de teorías, como procedimiento indirecto, ya que se concentran en la activación de situaciones de aprendizaje específicas, correspondientes al dominio que se pretende estudiar (Macchiarola, 2008; Pérez Echeverría, Mateos, Scheuer y Martín, 2006; Vilanova, García y Señoriño, 2007). Concretamente, este tipo de cuestionarios enfrentan a los sujetos a una serie de situaciones dilemáticas cotidianas, ofreciendo luego opciones de resolución para cada dilema. Dichas opciones se presentan mediante un discurso deliberativo y argumentativo que procura generar la adhesión de la persona que debe resolver los problemas planteados, seleccionando solamente una de las opciones propuestas. Las situaciones dilemáticas y sus opciones de resolución se elaboran a partir de constructos teóricos vinculados al dominio que se intenta estudiar y a las dimensiones que lo constituyen (Macchiarola, 2008).

En base a esto, se utilizó para la recolección de los datos empíricos un cuestionario de dilemas, que fue elaborado y revisado para el desarrollo de una investigación mayor y sometido a pruebas requeridas para estos instrumentos, las que mostraron su validez y confiabilidad (Boatto, Vélez y Bono, 2011). El cuestionario se conforma por 12 situaciones dilemáticas, contextualizadas en tres escenarios educativos. Éstos atienden a situaciones prácticas que enfrentan cotidianamente los sujetos y se denominan: 1. Leer para escribir con otros; 2. 
Leer para hacer una presentación oral individual y 3. Leer para ser evaluado. A su vez, para cada uno de estos escenarios se plantearon cuatro dilemas que atienden a cuatro categorías respectivamente: concepto, condiciones, procesos y resultados ${ }^{2}$ de aprendizaje a partir de la lectura. Cada uno de estos dilemas presenta tres opciones de resolución, enmarcadas en las dimensiones consideradas para el instrumento: Teoría Reproductiva Directa, Teoría Interpretativa Interactiva y Teoría Constructiva Transaccional.

Estas dimensiones se definen en base a las teorías implícitas sobre el aprendizaje, desde las que se explican las relaciones entre sujeto y objeto de conocimiento: Teoría Directa, Teoría Interpretativa y Teoría Constructiva (Pozo, 2009; Pozo, Scheuer, Mateos y Pérez Echeverría, 2006). A su vez, cada una de estas teorías se vinculan conceptualmente con tres enfoques científicos de lectura, que atienden a las relaciones entre lector, texto y contexto: Lectura como Conjunto de Habilidades, Enfoque Interactivo y Enfoque Transaccional (Braslavsky, 2005; Dubois, 1987; Goodman, 1994; Rinaudo, 1999; Rosenblatt 1994) ${ }^{3}$. A continuación se presenta la Tabla 1, donde se definen las categorías del instrumento en función de las dimensiones de estudio.

\footnotetext{
${ }^{2}$ Pozo, Scheuer, Mateos y Pérez Echeverría (2006) plantean una definición de aprendizaje (concepto) y lo caracterizan como un sistema integrado por tres componentes: condiciones, procesos y resultados. Para estudiar las concepciones implícitas de aprendizaje a partir de la lectura, consideramos al concepto y a estos componentes como catego- rías.

${ }^{3}$ Se apela a las teorías científicas como marco de referencia inicial para el análisis de las teorías implícitas (Correa y Camacho, 1993).
} 


\section{Tabla 1. Dimensiones y categorías del cuestionario de dilemas ${ }^{4}$}

\begin{tabular}{|c|c|c|c|}
\hline$\overbrace{\text { Categorías }}^{\text {Dimensiones }}$ & Teoría Reproductiva Directa & Teoría Interpretativa Interactiva & Teoría Constructiva Transaccional \\
\hline $\begin{array}{l}\text { Concepto de aprendizaje } \\
\text { Tipo de entidad que se le } \\
\text { otorga al conocimiento. } \\
\text { ¿Qué es aprender? }\end{array}$ & $\begin{array}{l}\text { Reproducción literal de lo leído: aprender } \\
\text { es expresar exactamente lo que el texto } \\
\text { dice. Se prioriza un único resultado correc- } \\
\text { to. }\end{array}$ & $\begin{array}{l}\text { Interpretación de la/s idea/s del autor: apren- } \\
\text { der es expresar lo que dice el texto, en función } \\
\text { de los procesos desarrollados para ello. } \\
\text { Se prioriza un resultado correcto, pero éste se } \\
\text { considera sujeto al procedimiento de lectura, y } \\
\text { por lo tanto no se produce una reproducción } \\
\text { literal de lo leído. }\end{array}$ & $\begin{array}{l}\text { Construcción de un significado personal del texto: } \\
\text { aprender es recrear el texto. } \\
\text { Se prioriza la atención a características del lector, texto } \\
\text { y contexto; considerando que la transacción entre estos } \\
\text { factores posibilita distintas construcciones de un mismo } \\
\text { texto, por lo que no habría un único resultado posible } \\
\text { sino un perspectivismo en la interpretación. }\end{array}$ \\
\hline $\begin{array}{l}\text { Condiciones de aprendi- } \\
\text { zaje } \\
\text { Factores del lector, texto y/o } \\
\text { contexto involucrados en el } \\
\text { aprendizaje. Así como el } \\
\text { modo en que el aprendiz } \\
\text { considera que operan en él. } \\
\text { ¿Qué factores inciden en el } \\
\text { aprendizaje? } \\
\text { ¿Cómo operan esos factores? }\end{array}$ & $\begin{array}{l}\text { Presencia de factores no vinculados, rela- } \\
\text { tivos al lector, texto y/o ambiente físico. } \\
\text { Factores que operan como determinantes. }\end{array}$ & $\begin{array}{l}\text { Factores referentes al lector y al texto, vincu- } \\
\text { lados. } \\
\text { Factores en los que interviene procesos menta- } \\
\text { les y afectivos. }\end{array}$ & $\begin{array}{l}\text { Interdependencia entre factores relativos al lector, texto } \\
\text { y contexto. } \\
\text { Factores en los que intervienen procesos mentales y } \\
\text { afectivos que provocan cambios en el aprendizaje. }\end{array}$ \\
\hline $\begin{array}{l}\text { Proceso de aprendizaje } \\
\text { Actividad desarrollada para } \\
\text { poder aprender. } \\
\text { ¿Cómo se aprende? }\end{array}$ & $\begin{array}{l}\text { Actividad observable - acto mecánico de } \\
\text { lectura. } \\
\text { (Incorporación de la información presente } \\
\text { en el texto, práctica y repetición a los } \\
\text { fines de la memorización). }\end{array}$ & $\begin{array}{l}\text { Actividad interpretativa. } \\
\text { (Incorporación de información presente en el } \\
\text { texto leído, mediante procesos de selección, } \\
\text { organización, memorización, control de lo } \\
\text { memorizado, autocorrección, etc.). }\end{array}$ & $\begin{array}{l}\text { Actividad constructiva. } \\
\text { (Construcción y reconstrucción del conocimiento, en } \\
\text { base a la reflexión, indagación, mirada crítica, etc. } \\
\text { frente al texto). }\end{array}$ \\
\hline $\begin{array}{l}\text { Resultado de aprendizaje } \\
\text { Producto del aprendizaje. } \\
\text { ¿Qué se aprende? }\end{array}$ & $\begin{array}{l}\text { Se aprende lo que el texto dice: los conte- } \\
\text { nidos literales expuestos en el texto. }\end{array}$ & $\begin{array}{l}\text { Se aprende lo que el texto representa: los } \\
\text { conocimientos están presentes en el texto y el } \\
\text { lector debe representárselos a sí mismo para } \\
\text { poder aprenderlos. }\end{array}$ & $\begin{array}{l}\text { Se aprende la representación que el lector puede hacer } \\
\text { del texto: el lector reelabora los contenidos para apren- } \\
\text { derlos, construyendo un texto paralelo. }\end{array}$ \\
\hline
\end{tabular}

${ }^{4}$ Las dimensiones se definieron en base a las teorías implícitas de aprendizaje: Directa, Interpretativa y Constructiva (Rodrigo, Rodríguez yMarrero, 1993; Pozo yScheuer, 1999; Pozo, Scheuer, Mateos yPérez Echeverría, 2006); vinculándolas a las teorías científicas de la lectura: Lectura como Conjunto de Habilidades, Enfoque Interactivo y Enfoque Transaccional (Braslavsky, 2005; Dubois, 1987; Goodman, 1994; Rosenblatt 1994; Rinaudo, 1999). 


\section{Diseño y Procedimiento}

El estudio asume carácter no experimental, en tanto se describen y analizan los fenómenos en su contexto natural sin manipular ninguna variable, y transeccional descriptivo, debido a que se recopilan los datos en un único momento y se indaga y analiza la incidencia e interacción de las modalidades de la variable estudiada en un grupo de sujetos (Hernández Sampieri, Fernández Collado y Bapista Lucio, 2006). La variable de estudio está referida a las concepciones de aprendizaje a partir de la lectura de textos académicos de estudiantes que ingresan a la universidad, en la situación de leer para escribir junto a pares.

Para responder al objetivo específico de describir y analizar las concepciones de aprendizaje a partir de la lectura de textos académicos que mantienen los estudiantes universitarios, que ingresan a ciencias de la educación, en la situación de leer para escribir junto a pares, se consideraron las situaciones del cuestionario de dilemas que se enmarcan en el escenario 'leer para escribir con otros' y las opciones de resolución de dichas situaciones seleccionadas por los alumnos. Se procedió a analizar las respuestas de los sujetos mediante: a) estadísticos descriptivos y b) análisis de correspondencias múltiples.

\section{Análisis de datos}

Se administró el cuestionario a los 118 estudiantes universitarios. Luego, se procedió a la carga y procesamiento de los datos mediante el paquete estadístico SPSS y se realizaron análisis descriptivos y de correspondencias múltiples.

\section{Resultados}

Descriptivos

El procedimiento de análisis de estadísticos descriptivos permitió conocer de manera descriptiva las concepciones de aprendizaje a partir de la lectura de textos académicos de los estudiantes, en la situación de leer para escribir junto a sus pares, atendiendo a los componentes (categorías) que integran dichas concepciones. A continuación se presenta la Figura 1, que muestra las frecuencias de las dimensiones (Teoría Reproductiva Directa, Teoría Interpretativa Interactiva y Teoría Constructiva Transaccional) en cada una de las categorías estudiadas (concepto, condiciones, proceso y resultados de aprendizaje a partir de la lectura) en el escenario 'leer para escribir con otros'. 


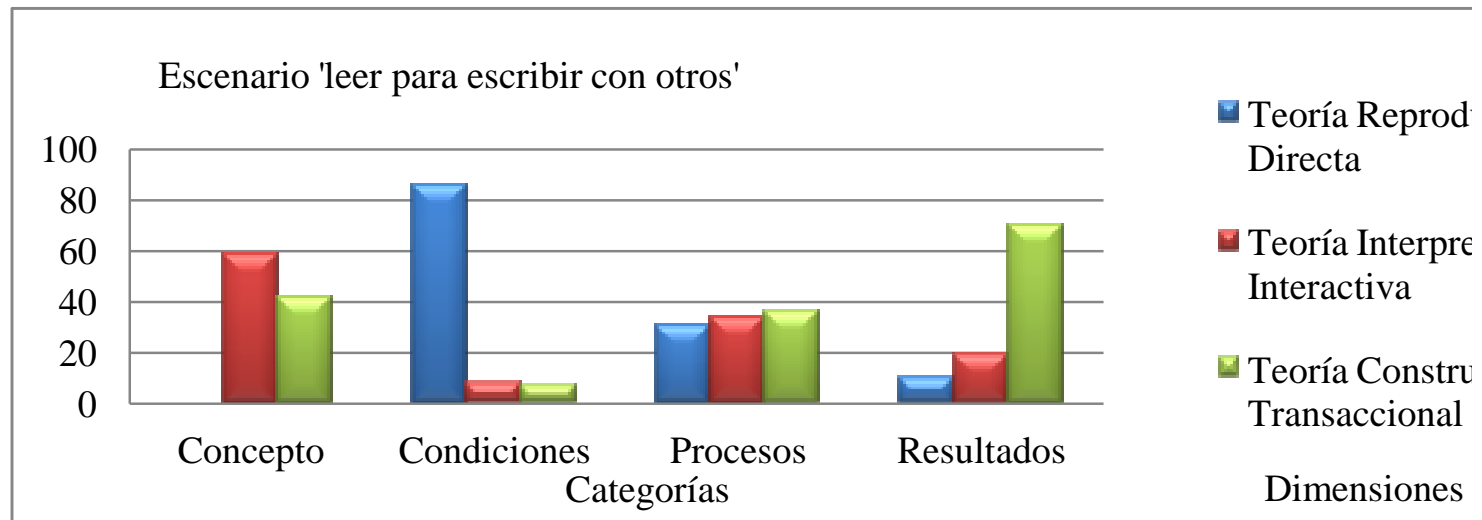

Figura 1. Dimensiones y categorías de las concepciones de aprendizaje a partir de la lectura de textos académicos.

1) Para la categoría concepto de aprendizaje a partir de la lectura, más de la mitad de los alumnos $(58,5 \%)$ eligieron la opción donde el concepto se entiende como la 'interpretación de las ideas del autor', pero ajustando tal interpretación a lo que el texto dice, asociándose de este modo a una Teoría Interpretativa Interactiva, tal como se expresa en la opción propuesta para resolver el dilema:

"Hay que ver qué cosas se marcaron en los libros como importantes y organizar el trabajo a partir de ahí, porque no se puede poner todo lo que dicen los autores sino lo más importante”.

Para estos alumnos, aprender sería expresar lo que dice el texto, en función de los procesos desarrollados para ello, priorizándose un resultado correcto, pero considerándolo ajustado al texto. El resto de los estudiantes $(41,5 \%)$ eligió la opción donde el concepto de aprendizaje se define como la 'construcción de un significado personal del texto', idea vinculada a una Teoría Constructiva Transaccional. El enunciado que representa esta opción se presenta en el cuestionario de la siguiente manera:

"Deberíamos revisar de nuevo los libros y de todo lo que teníamos ver que es lo más importante para nosotros, así podemos proponer ideas nuevas teniendo en cuenta lo que dicen los autores”.

Para estos alumnos, aprender sería recrear el texto, atendiendo a las características del lector, del texto y del contexto; considerando que la transacción entre estos factores posibilitaría distintas construcciones, por lo que no habría un único resultado posible sino un perspectivismo en la interpretación. En ningún caso los estudiantes seleccionaron la opción que explica al concepto de aprendizaje como la 'reproducción literal de lo leído', donde aprender 
sería expresar exactamente lo que el texto dice, priorizándose un único resultado correcto; idea ésta que se vincula a una Teoría Reproductiva Directa.

2) Respecto a la categoría condiciones de aprendizaje a partir de la lectura, la mayoría de los sujetos $(85,6 \%)$ eligió la opción donde las condiciones relativas al lector, texto y/o contexto operan como determinantes en el aprendizaje y aparecen como factores desvinculados entre sí. Estas ideas pueden asociarse a una Teoría Reproductiva Directa, tal como se expresa en la opción propuesta para resolver el dilema:

"Para poder aprender de la fotocopia lo más importante es tener paciencia y ponerse, buscar un lugar cómodo, tranquilo y ponerle ganas”.

Es poco significativa la cantidad de alumnos que entiende a las condiciones como factores del lector y el texto, que se vinculan e inciden en el aprendizaje (7,6\%); o como factores interdependientes relativos al lector, texto y contexto, que provocan cambios en el aprendizaje $(6,8 \%)$. La primera idea se asocia a una Teoría Interpretativa Interactiva y la segunda a una Teoría Constructiva Transaccional.

3) En la categoría procesos de aprendizaje a partir de la lectura, los porcentajes de elección de opciones vinculadas a cada una de las teorías implícitas de aprendizaje son similares. El 30,5\% de los alumnos seleccionó la opción donde los procesos se entienden como una 'actividad observable', asociada a una Teoría Reproductiva Directa. En la opción se expresa:

"Ir leyendo por partes, buscar en el diccionario las palabras que no se entienden o marcarlas en el libro para preguntárselas al profesor en la clase. También es necesario subrayar y copiar las partes más importantes”.

Estos estudiantes advierten la necesidad de poner en marcha diversas acciones, sin embargo no reconocen los procesos de pensamiento que darían lugar a las transformaciones del texto en interacción con el conocimiento previo del lector. Las acciones seleccionadas son aquéllas que contribuyen a un mejor 'ajuste al texto'. Por otra parte, el 33,9\% de los alumnos eligió la opción en la que los procesos se entienden como una 'actividad interpretativa'. Esto se expresa del siguiente modo:

"Ver qué nos quiere decir el autor, prestar atención a las partes más importantes y relacionarlas con cosas ya conocidas, interpretar el significado de las palabras desconocidas y cuando algo no se entiende marcarlo en el libro para consultar al profesor en clase'. 
Concepciones de estudiantes que ingresan a la universidad sobre el aprendizaje mediado por la lectura, en contextos de escritura académica

Para los sujetos que eligieron esta opción, asociada a una Teoría Interpretativa Interactiva, la comprensión sería un proceso activo pero literal, en el sentido que lo plantea Braslavsky $(2005)^{5}$. Tratarían de interpretar la información presente en el texto leído mediante procesos de selección, memorización, reflexión y autocorrección.

Por último, el 35,6\% restante optó por la opción donde los procesos se entienden como una 'actividad constructiva'. Expresado esto del siguiente modo:

"Ponerse de acuerdo en cuál va a ser el objetivo del grupo y a medida que se lee ir viendo si lo que dice el libro ya es conocido, para poder ir relacionándolo con lo que se quiere aprender, deteniéndose ante las dificultades que se presenten, intentando superarlas, y en caso de no poder, preguntar en clase sobre lo que no se entiende".

Esta opción se enmarca en una Teoría Constructiva Transaccional. Aquí la comprensión sería activa, se produciría una construcción y reconstrucción del conocimiento, en base a la reflexión e indagación frente al texto.

4) En la categoría resultados de aprendizaje a partir de la lectura, los alumnos eligieron mayoritariamente $(70,3 \%)$ la opción donde el resultado implicaría la 'representación que el lector puede hacer del texto', idea vinculada a una Teoría Constructiva Transaccional. El enunciado de la opción elegida es el siguiente:

"Se aprendieron los temas y también a reflexionar sobre lo que decían los tex-

tos, haciendo relaciones entre ellos y elaborando conocimientos nuevos”.

Desde esta perspectiva, el lector reelaboraría los contenidos para aprenderlos, construyendo un 'texto paralelo' ${ }^{6}$. Son escasas las elecciones por parte de los alumnos, que consideren que se aprende 'lo que el texto representa' (19,5\%) -los contenidos presentes en el texto pero representados por el lector- o 'lo que el texto dice' (10,2\%) -los contenidos literales expuestos en el texto-. Estas ideas se vinculan a una Teoría Interpretativa Interactiva y a una Teoría Reproductiva Directa respectivamente.

Resumiendo, el análisis descriptivo de los datos muestra que en la categoría concepto de aprendizaje a partir de la lectura, más de la mitad de los alumnos (58.5\%) entienden al

\footnotetext{
${ }^{5}$ La comprensión literal implica por parte del lector la intervención sobre el texto para comprender los planteos del autor, pero en búsqueda de una única idea posible (Braslavsky, 2005).

${ }^{6}$ El concepto de texto paralelo fue planteado por Goodman (1994) en relación a la reconstrucción que el lector hace del texto a partir de sus propósitos y conocimientos previos.
} 
concepto como la 'interpretación de las ideas del autor' y otro grupo algo menor (41.5\%) lo entiende como la 'construcción de un significado personal del texto'; ideas que se vinculan a las Teorías Interpretativa Interactiva y Constructiva Transaccional, respectivamente. Respecto a la categoría condiciones de aprendizaje a partir de la lectura, la mayoría de los estudiantes (85.6\%) asume que las condiciones relativas al lector, texto y/o contexto operan como determinantes en el aprendizaje y aparecen como factores desvinculados entre sí, estas ideas se relacionan a una Teoría Reproductiva Directa. En cambio, en la categoría procesos de aprendizaje a partir de la lectura, las elecciones se reparten de manera semejante entre la Teoría Reproductiva Directa, la Interpretativa Interactiva y la Constructiva Transaccional; un grupo de alumnos (30.5\%) entiende a los procesos como una 'actividad observable', vinculada a una Teoría Reproductiva Directa; otro grupo (33.9\%) como una 'actividad interpretativa', relacionada a una Teoría Interactiva Interpretativa y el resto de los alumnos (35.6\%) como una 'actividad constructiva', ligada a una Teoría Constructiva Transaccional. En la categoría resultados de aprendizaje a partir de la lectura, los alumnos conciben mayoritariamente $(70.3 \%)$ que el resultado implicaría la 'representación que el lector puede hacer del texto', idea que se relaciona a una Teoría Constructiva Transaccional.

Estos resultados muestran que las cuatro categorías estudiadas en conjunto no se corresponden con una única teoría implícita de aprendizaje, y que cada una de ellas se define de modo diferente, ya sea desde una teoría implícita preponderante o desde la coexistencia de dos o tres teorías implícitas. Avanzando en el análisis del modo en que se configuran estas cuatro categorías en conjunto, se presentan a continuación los resultados del análisis de correspondencias múltiples.

\section{Correspondencias múltiples}

El procedimiento de análisis de correspondencias múltiples ${ }^{7}$ permitió avanzar en el conocimiento de las configuraciones que asumen las diferentes categorías -concepto, condiciones, procesos y resultados- que conformarían las concepciones de aprendizaje estudiadas, tal como se muestra en la Figura 2.

\footnotetext{
${ }^{7}$ Análisis de homogeneidad con técnica de escalamiento óptimo, que representa mediante un modelo geométrico las medidas de las distancias entre variables, dando cuenta de la estructura implícita de la matriz de datos. Este análisis espacial multivariado permite conocer el patrón de agrupamiento de las categorías, ya que reúne variables por semejanzas y oposición y realiza nuevos agrupamientos que se conforman a partir de la combinación lineal de las variables iniciales (Visauta y Martori, 2003).
} 


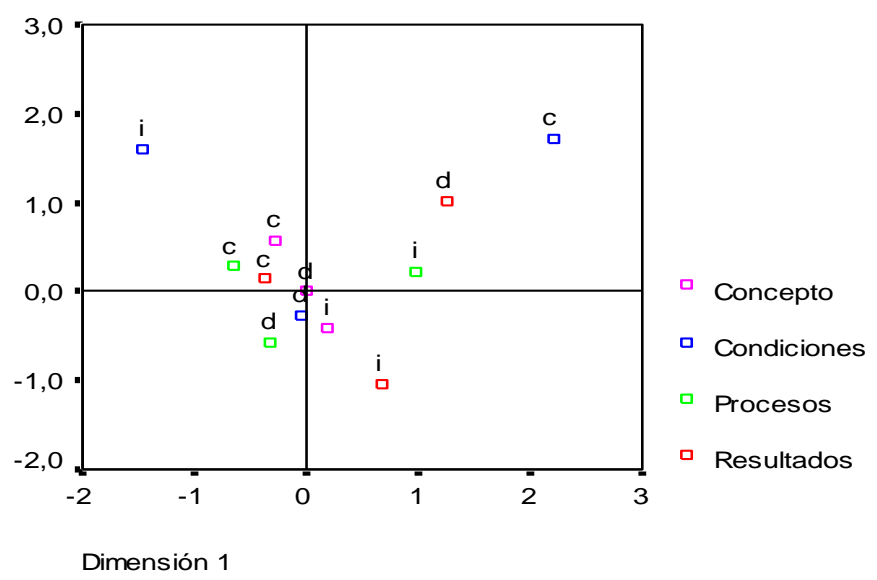

Nota: d: Teoría Reproductiva Directa - i: Teoría Interpretativa Interactiva - c: Teoría Constructiva Transaccional.

Figura 2. Configuraciones de concepciones de aprendizaje a partir de la lectura: leer para escribir junto a pares.

La Figura 2 muestra cuatro configuraciones en forma de agrupamientos:

Primera configuración. En el cuadrante superior derecho el agrupamiento conformado se caracteriza por la presencia de distintas categorías correspondientes a las concepciones de aprendizaje a partir de la lectura, cuando se lee para escribir junto a pares, definidas desde diferentes dimensiones. Las condiciones de aprendizaje a partir de la lectura aparecen definidas desde la 'Teoría Constructiva Transaccional', los procesos de aprendizaje a partir de la lectura desde la 'Teoría Interpretativa Interactiva' y los resultados de aprendizaje a partir de la lectura desde la 'Teoría Reproductiva Directa'. La categoría concepto de aprendizaje a partir de la lectura no aparece en este grupo. Este primer agrupamiento se muestra heterogéneo e incompleto teóricamente, si se lo compara con la estructura lógica conceptual de las distintas teorías implícitas de aprendizaje.

Segunda configuración. En el cuadrante superior izquierdo se observa un agrupamiento cuyas características predominantes corresponden a las dimensiones 'Teoría Constructiva Transaccional' y en menor medida a la 'Teoría Interpretativa Interactiva'. Específicamente, aparecen agrupados el concepto, los procesos y los resultados de aprendizaje a partir de la lectura, cuando se lee para escribir junto a pares, definidos desde una Teoría Constructiva Transaccional, mientras que las condiciones aparecen desde una Teoría Interpretativa Interactiva. Este segundo agrupamiento, a diferencia del anterior, presenta mayor completud y menor 
heterogeneidad en términos teóricos, en comparación con las teorías implícitas de aprendizaje. Mayor completud porque se conforma en base a las cuatro categorías (concepto, condiciones, procesos y resultados de aprendizaje a partir de la lectura). Menor heterogeneidad porque se define para tres categorías desde la Teoría Constructiva Transaccional y sólo en una categoría desde la Teoría Interpretativa Interactiva.

Tercera configuración. En el cuadrante inferior izquierdo se observa un agrupamiento caracterizado por la dimensión 'Teoría Reproductiva Directa', que contiene a las categorías condiciones y procesos de aprendizaje a partir de la lectura. Las categorías concepto y resultados de aprendizaje a partir de la lectura no aparecen aquí. Este tercer agrupamiento se muestra homogéneo pero incompleto en términos teóricos, si se lo compara con las teorías implícitas de aprendizaje.

Cuarta configuración. El cuadrante inferior derecho muestra un agrupamiento definido desde la dimensión 'Teoría Interpretativa Interactiva', que contiene a las categorías concepto y resultados de aprendizaje a partir de la lectura. Por lo tanto este cuarto grupo también es homogéneo pero incompleto en términos teóricos, en comparación a las teorías implícitas de aprendizaje, ya que no aparecen aquí las categorías condiciones y procesos de aprendizaje a partir de la lectura.

En síntesis, las configuraciones que asumen las categorías que conformas las concepciones estudiadas se caracterizan de acuerdo a una de las dos siguientes tendencias: a) completud pero heterogeneidad en términos teóricos: las configuraciones se constituyen por todas o la mayor parte de las categorías, pero en base a diferentes teorías implícitas; b) homogeneidad pero incompletud en términos teóricos: las configuraciones se definen desde una misma teoría implícita, pero son insuficientes en cuanto a su constitución categórica.

\section{Discusión y conclusiones}

El objetivo de este trabajo, como se señaló inicialmente, ha sido el de describir y analizar las concepciones de aprendizaje a partir de la lectura de textos académicos, en la situación de leer para escribir junto a pares, que mantienen los estudiantes que ingresan a la universidad, específicamente a ciencias de la educación.

Los resultados obtenidos del primer análisis realizado muestran que las concepciones implícitas de aprendizaje a partir de la lectura que mantienen los estudiantes, en la situación de escribir junto a pares, serían inconsistentes en relación a la manera en la que se configura 
cada una de las teorías implícitas; en tanto los datos empíricos también informan que no se podría validar completamente el constructo teórico estudiado. Entendemos que estos resultados admiten la reconsideración del término 'teoría' para hacer referencia a las concepciones implícitas. En este sentido, acordamos con Castorina (2010) respecto de la discusión sobre el uso del constructo 'teoría' para dar cuenta de las concepciones implícitas. Este autor, recuperando planteos de Bruner (1990), considera que tales concepciones se constituyen por "saberes narrativos" que no se estructuran desde una lógica conceptual, como lo hacen las teorías científicas. Desde esta perspectiva, las concepciones implícitas o de sentido común se presentan de manera incoherente o inconsistente debido a su naturaleza tácita y por tanto incomunicable; implican presuposiciones generales sobre distintos tipos de relaciones que explicarían determinados acontecimientos, pero éstas no son conscientes para los sujetos. En cambio, las concepciones científicas necesariamente deben explicarse en base a un lenguaje o sistema de representación compartido por una comunidad científica, ante lo que deben mostrar coherencia y consistencia (Kaplan, 1997; Pozo, Scheuer, Mateos y Pérez Echeverría, 2006).

Así mismo, Wellman (1990) expone que una teoría, para constituirse como tal, debe contener tres rasgos: 1 . ser un conocimiento coherente, una red interrelacionada de constructos y términos, donde los conceptos se entrelazan y cada uno apoya al resto; 2 . ser distinciones o compromisos ontológicos específicos, destacar rasgos relevantes en diferentes campos, permitir la diferenciación de los fenómenos en base a distintos tipos de entidades y procesos y; 3. ser un marco causal-explicativo que permita justificar, comprender y formular predicciones sobre los fenómenos de un campo. En este mismo sentido, Castorina define a las teorías como "sistemas interpretativos de la realidad, constituidos por un conjunto de afirmaciones, organizadas entre sí, que permiten explicar y predecir fenómenos en distintos campos cognoscitivos" (2007, p. 209).

Partiendo de estas conceptualizaciones, consideramos que la configuración del primer agrupamiento (primer cuadrante) no se corresponde con la conformación de una 'teoría'. Este agrupamiento es el que más se aleja de los constructos teóricos que explican las diferentes teorías implícitas de aprendizaje de los sujetos, ya que se muestra totalmente mixto, definiéndose desde las tres teorías implícitas (Directa, Interpretativa y Constructiva). Además, es insuficiente categóricamente para constituirse en una teoría, porque se define desde las condiciones, proceso y resultados de aprendizaje a partir de la lectura, pero la categoría concepto de aprendizaje no aparece en este grupo. 
En cambio, la segunda configuración (segundo cuadrante) responde al criterio de completud, ya que aparece conformada desde las cuatro categorías estudiadas, y es la configuración que menor heterogeneidad presenta, porque se define en gran medida desde la Teoría Constructiva -específicamente en las categorías concepto, procesos y resultados de aprendizaje a partir de la lectura-. Aunque, se hace presente también un rasgo de la Teoría Interpretativa -que se manifiesta en la categoría condiciones de aprendizaje a partir de la lectura-, lo que no le permite a esta configuración constituirse como un agrupamiento puramente homogéneo que se defina desde una teoría en particular.

Este agrupamiento podría entenderse desde las investigaciones y postulados teóricos que se ocupan de las concepciones de aprendizaje entendidas como teorías implícitas (Pozo, 2009; Pozo, Scheuer, Mateos y Pérez Echeverría, 2006; Scheuer y Pozo, 2006), ya que desde allí se plantea que entre la Teoría Interpretativa y la Teoría Constructiva existirían concepciones intermedias, que se van generando en el transcurso del proceso de cambio representacional que podría experimentar el sujeto (Scheuer y Pozo, 2006).

Finalmente, las configuraciones de los últimos dos agrupamientos (tercer y cuarto cuadrante) aparecen definidas desde una única teoría -la Directa en el tercer cuadrante y la Interpretativa en el cuarto- lo que permitiría pensar en una mayor vinculación con los desarrollos teóricos respecto de las concepciones implícitas de aprendizaje. Sin embargo, en ambos casos la constitución categórica no es suficiente para dar cuenta de la conformación de una 'teoría', ya que en el tercer cuadrante el agrupamiento aparece definido sólo desde las condiciones y procesos de aprendizaje a partir de la lectura, y en el cuarto cuadrante aparece definido sólo desde el concepto y los resultados de aprendizaje a partir de la lectura.

Por otro lado, es interesante destacar que los resultados muestran que además de la cuarta configuración, definida principalmente desde la Teoría Interpretativa, cuya presencia sería preponderante en las concepciones implícitas de los estudiantes de nivel superior (así como de otros niveles educativos); aparecen otras configuraciones definidas desde la Teoría Directa (tercera) y desde la Teoría Constructiva (segunda), cuya presencia se supone secundaria o menor, desde los estudios en el campo (Scheuer y Pozo, 2006).

En síntesis, los resultados hallados en este estudio advierten y dejan planteada la necesidad de repensar la pertinencia del uso del término 'teoría' para hacer referencia a las concepciones implícitas de aprendizaje, que mantienen los estudiantes que ingresan a la universi- 
dad, cuando leen para escribir junto a pares; muestran además que el modelo de las teorías implícitas se valida parcialmente, en la situación particular estudiada en esta investigación.

\section{Agradecimientos}

Este trabajo forma parte de una investigación más amplia enfocada en el estudio de las concepciones sobre el aprendizaje a partir de la lectura de textos académicos, que mantienen los estudiantes que ingresan a la universidad. El Programa de investigación del que se desprende este estudio es: La lectura, la escritura y el interés por aprender en la universidad: la producción de conocimientos académico-profesionales. 2012-2014. Aprobado y subsidiado por la por la Secretaría de Ciencia y Técnica de la Universidad Nacional de Río Cuarto, Argentina.

\section{Referencias}

Atkinson, T. y Claxton, G. (2000) El profesor intuitivo. Barcelona: Octaedro.

Boatto, Y., G. Vélez y A. Bono (2011) Construcción de un cuestionario de dilemas para indagar las concepciones sobre el aprendizaje a partir de la lectura en ingresantes universitarios. Summa Psicológica UST, Revista de Psicología de la Universidad Santo Tomás de Chile. ISSN 0718-0446.

Bosch, M.B. y Scheuer, N. (2006) Resumir para estudiar: concepciones de estudiantes en primer año de la universidad. En J.I. Pozo; Scheuer, N.; Pérez Echeverrría, M.P.; Mateos, M.; Martín, E. y De la Cruz, M. (Eds.), Nuevas formas de pensar la enseñanza y el aprendizaje. Las concepciones de profesores y alumnos (pp. 341-353). Barcelona: GRAÒ

Braslavsky, B. (2005) La comprensión del lenguaje escrito. En B. Braslavsky, Enseñar a entender lo que se lee. La alfabetización en la familia y en la escuela (pp. 48-54). México: Fondo de Cultura Económica.

Bruner, J. (1990) Actos de significado. Más allá de la revolución cognitiva. Madrid: Alianza.

Carlino, P. (2005) Escribir, leer y aprender en la universidad. Una introducción a la alfabetización académica. Buenos Aires: Fondo de de Cultura Económica.

Castelló, M. (2007) El proceso de composición de textos académicos. En M. Castelló (coord.), Escribir y comunicarse en contextos científicos y académicos (pp. 47-81). Barcelona: Graó.

Castelló, M. (2009) Aprender a escribir textos académicos: ¿Copistas, escribas, compiladores o escritores? En Pozo J. I. y M. del P. Pérez Echeverría (coords.), Psicología del 
aprendizaje universitario: La formación en competencias (pp. 120-127). Madrid: Morata.

Castorina, J.A. (2010) Algunos problemas filosóficos de las teorías del cambio conceptual. Seminario Internacional de Cambio Conceptual. Buenos Aires: FLACSO.

Castorina, J.A.; Barreiro, A. y A.G. Toscano (2007/2005) Dos versiones del sentido común: las Teorías Implícitas y las representaciones sociales. En J.A. Castorina (coord.), Construcción conceptual y representaciones sociales. El conocimiento de la sociedad (pp. 205-238). Argentina: Miño y Dávila.

Correa, A. y J. Camacho (1993) Diseño de una metodología para el estudio de las teorías implícitas. En M. J. Rodrigo; A. Rodríguez y J. Marrero (1993), Las teorías implícitas. Una aproximación al conocimiento cotidiano (pp. 123-166). Madrid: Visor.

Dubois, M. E. (1987) El Proceso de Lectura. De la Teoría a la Práctica. Buenos Aires: Aique.

Fernández G., Izuzquiza M.V., Ballester M.A., Marrón, M.P., Eizaguirre, M.D., y Zanotti, F. (2010) Leer y escribir para aprender en los primeros años de la universidad. Lectura y Vida, 31 (3), 62-71.

Fernández, G. y Carlino, P. (2010) ¿En qué se diferencian las prácticas de lectura y escritura de la universidad y las de la escuela secundaria? Lectura y Vida, 31 (3), 6-19.

Goodman, K. (1994). La lectura, la escritura y los textos escritos: una perspectiva transaccional y sociopsicolingüística. Textos en contexto (2). Los procesos de lectura y escritura. Lectura y Vida. Buenos Aires. 11-68.

Hernández Sampieri, R.; C. Fernández Collado y P. Bapista Lucio (2006) Concepción o elección del diseño de investigación. En R. Hernández Sampieri, C. Fernández Collado y P. Bapista Lucio, Metodología de la investigación (pp. 157-231). México: McGrawHill Interamericana.

Kaplan, C. (1997) Planteamiento del problema. En Kaplan, C., La inteligencia escolarizada. Un estudio de las representaciones sociales de los maestros sobre la inteligencia de los alumnos y su eficacia simbólica (pp. 21-48). Buenos Aires: Miño y Dávila. Colección Aprendizaje Subjetividad.

Macchiarola, V. (2008) Metodología. En V. Macchiarola, Concepciones sobre el planeamiento institucional (pp. 89-116). Río Cuarto: Editorial Universidad Nacional de Río Cuarto.

Pérez Echeverría, M. P.; M. Mateos y N. Scheuer (2006) Enfoques en el estudio de las concepciones sobre el aprendizaje y la enseñanza. En J.I. Pozo; N. Scheuer; M.P. Pérez- 
Concepciones de estudiantes que ingresan a la universidad sobre el aprendizaje mediado por la lectura, en contextos de escritura académica

Echeverrría, M. Mateos, E. Martín y M. De la Cruz (Eds.), Nuevas formas de pensar la enseñanza y el aprendizaje. Las concepciones de profesores y alumnos (pp. 55-89). Barcelona: GRAÒ.

Pérez-Echeverría, M. P.; J. I. Pozo, A. Pecharromán, J. Cervi y P. Martínez (2006) Las concepciones de los profesores de educación secundaria sobre el aprendizaje y la enseñanza. En J.I. Pozo; N. Scheuer; M.P. Pérez-Echeverrría, M. Mateos, E. Martín y M. De la Cruz (Eds.), Nuevas formas de pensar la enseñanza y el aprendizaje. Las concepciones de profesores y alumnos (pp. 289-302). Barcelona: GRAÒ.

Pozo J. I. (2009) Adquirir una concepción compleja del conocimiento: Creencias epistemológicas y concepciones de aprendizaje. En Pozo J. I. y M. del P. Pérez Echeverría (coords.), Psicología del aprendizaje universitario: La formación en competencias (pp. 70-80). Madrid: Morata.

Pozo J.I. y Pérez Echeverría, M. del P. (2009) Psicología del aprendizaje universitario: La formación en competencias. Madrid: Morata.

Pozo, J. I. y N. Scheuer (1999) Las concepciones sobre el aprendizaje como teorías implícitas. En J. I. Pozo y C. Monereo (Coords.) El aprendizaje estratégico (pp. 87-108). Madrid: Santillana.

Pozo, J. I., N. Scheuer, M. Mateos y M. P. Pérez Echeverría (2006) Las Teorías Implícitas sobre el aprendizaje y la enseñanza. En J.I. Pozo; N. Scheuer; M.P. Pérez-Echeverrría, M. Mateos, E. Martín y M. De la Cruz (Eds.), Nuevas formas de pensar la enseñanza y el aprendizaje. Las concepciones de profesores y alumnos (pp. 95-127). Barcelona: GRAÒ.

Pozo, J.I, N. Scheuer, M.P. Pérez Echeverría, M. Mateos, E. Martín y M. de la Cruz (Eds.) (2006) Nuevas formas de pensar la enseñanza y el aprendizaje. Las concepciones de profesores y alumnos. Barcelona: GRAÒ.

Pozo, J.I. (2001) Humana mente. El mundo, la conciencia y la carne. Madrid. Morata

Rinaudo, M. C. (1999) La naturaleza del proceso de comprensión de textos. En M. C. Rinaudo, Comprensión del texto escrito (pp. 13-24). Río Cuarto: Editorial de la Fundación Universidad Nacional de Río Cuarto.

Rodrigo, M. J.; A. Rodríguez y J. Marrero (1993) Las teorías implícitas. Una aproximación al conocimiento cotidiano. Madrid: Visor.

Rosenblatt, L. M. (1994) La teoría transaccional de la lectura y la escritura. Textos en contexto $\mathrm{N}^{\circ}$ I. Los procesos de lectura y escritura. Lectura y Vida. Buenos Aires. 1996. 16-71. 
Scheuer, M. y Pozo, J.I (2006) ¿Qué cambia en las Teorías Implícitas sobre el aprendizaje y la enseñanza? Dimensiones y procesos de cambio representacional. En J.I. Pozo; N. Scheuer; M.P. Pérez-Echeverrría, M. Mateos, E. Martín y M. De la Cruz (Eds.), Nuevas formas de pensar la enseñanza y el aprendizaje. Las concepciones de profesores y alumnos (pp. 375-402). Barcelona: GRAÒ.

Vélez, G. (2006) Las autobiografías lectoras como autobiografías de aprendizaje. En J.I. Pozo; N. Scheuer; M.P. Pérez-Echeverrría, M. Mateos, E. Martín y M. De la Cruz (Eds.), Nuevas formas de pensar la enseñanza y el aprendizaje. Las concepciones de profesores y alumnos (pp. 307-319). Barcelona: GRAÒ.

Vilanova S. L., M. B. García y O. Señoriño (2007) Concepciones acerca del aprendizaje: diseño y validación de un cuestionario para profesores en formación. Revista Electrónica de Investigación Educativa, 9 (2). Consultado el 15 de marzo de 2009, en http://redie.uabc.mx/vo19no2/contenido-vilanova.html

Visauta, B. y J. Martori (2003). Análisis estadístico con SPSS para Windows. España: Ed. McGraw Hill.

Wellman, H. (1990) Infancia. Teorías y pensamiento. Introducción. En Desarrollo de la teoría del pensamiento en los niños (pp. 19-29). Bilbao: Descleé de Brouer, 1995. 